Transcontinentales

continentales

Sociétés, idéologies, système mondial

$4 \mid 2007$

Amérique latine

\title{
Amérique latine : gauches et mondialisation
}

Jean-Luc Racine

\section{OpenEdition}

\section{Journals}

Édition électronique

URL : http://journals.openedition.org/transcontinentales/580

DOI : 10.4000/transcontinentales.580

ISBN : 978-2-7351-1565-5

ISSN : 1775-397X

Éditeur

Editions de la maison des sciences de l'homme

Édition imprimée

Date de publication : 1 juin 2007

Pagination : 5-13

ISBN : 978-2-200-92396-9

ISSN : $1950-1684$

\section{Référence électronique}

Jean-Luc Racine, «Amérique latine : gauches et mondialisation », Transcontinentales [En ligne], 4

2007, document 1, mis en ligne le 27 avril 2011, consulté le 25 septembre 2020. URL : http://

journals.openedition.org/transcontinentales/580 ; DOI : https://doi.org/10.4000/transcontinentales. 580 


\section{Amérique latine : gauches et mondialisation}

Fait marquant des années 2005-2006, les victoires multiples de la gauche latinoaméricaine (des gauches, dira-t-on plus loin) appellent à être replacées dans le contexte historique qui est le leur. Au fil du xx siècle, l'Amérique latine a certes connu des mouvements révolutionnaires et des gouvernements socialistes. Mais les coups d'État militaires furent récurrents, et l'ombre américaine toujours pesante, Washington cherchant à protéger les intérêts des États-Unis dans "l'arrière-cour» définie par la doctrine Monroe dès 1823. À l'heure où l'Amérique latine opérait la première décolonisation de l'histoire - après Haïti en 1804, seize pays latinoaméricains, dont les plus grands d'entre eux, accédèrent à l'indépendance entre 1810 et 1825 - les États-Unis entendaient bien affirmer leur zone d'influence déterminante au sud de leur frontière, portée au Rio Grande en 1848. Cette hégémonie américaine, contrebalancée au XIX siècle par la prégnance persistante des influences européennes, s'affirmera pleinement après la première guerre mondiale.

Tout au long du $\mathrm{xx}^{e}$ siècle se succédèrent en effet des interventions américaines, directes ou indirectes, et furent proclamés des programmes ambitieux, telle l'Alliance pour le progrès de John F. Kennedy en 1961, ou l'Initiative pour les Amériques de George H. Bush père en 1990. Nombreuses en Amérique centrale, les interventions de Washington furent moins fréquentes en Amérique du Sud. Mais l'échec du débarquement anticastriste de la Baie des cochons en 1961, puis le blocus de Cuba, le coup d'État réussi contre Salvador Allende, au Chili en 1973, et le soutien durable à la contra anti-sandiniste du Nicaragua dans les années 1980 laissèrent, dans toute l'Amérique latine, des traces indélébiles dépassant les seules logiques de la guerre froide. L'opération «Condor», associant les services spéciaux du Chili, du Brésil, de l'Argentine, de la Bolivie, du Paraguay et de l'Uruguay, dans les années 1970, symbolisera les côtés les plus noirs de la lutte "anti-subversive» menée par des "escadrons de la mort». Patte de la CIA ou pas, les dictatures militaires sud-américaines bénéficièrent de l'appui, ouvert ou discret, de Washington, hantée par le spectre communiste incarné de longue date par un Fidel Castro aujourd'hui finissant et ravivé, sur un autre mode, par un Hugo Chávez jouant de l'arme pétrolière pour financer sa «révolution bolivarienne».

Autres temps, autres mœurs? Curieusement, les néo-conservateurs de GeorgeW. Bush n'auront pas cherché à endiguer efficacement la récente montée de la gauche latinoaméricaine. Certes, Washington est soupçonnée de connivence avec les militaires 
ayant tenté un coup d'État avorté contre Chávez en avril 2002, mais on a connu les États-Unis plus réactifs face à l'anti-américanisme sans nuance dans leur traditionnelle zone d'influence. En arrivant au pouvoir en 2001, GeorgeW. Bush avait annoncé son "engagement total» envers l'Amérique latine. En mars 2007, au moment du voyage présidentiel conduit pour contrer l'influence grandissante de Chávez, un éditorial du Boston Globe constatait : "Au lieu de rabâcher les discours sur l'intervention militaire contre la drogue (en Colombie) et sur les accords de libre-échange qui ne font apparemment qu'accroître pauvreté et inégalités, Bush devrait porter attention aux besoins spécifiques des pays qu'il va visiter [...]. Le meilleur moyen pour Bush de contenir la vague Chávez est de réparer une part des dommages accumulés au fil de six années de négligence ${ }^{1}$.»

Si négligence il y a, comment l'expliquer? Par l'extinction de la plupart des guérillas qui ont marqué le sous-continent? Quarante ans après la capture et l'exécution de Che Guevara en Bolivie, le Sentier lumineux péruvien n'est plus que l'ombre de lui-même, et l'Armée zapatiste de libération nationale, au Mexique, a abandonné la lutte armée ${ }^{2}$. Le dernier grand foyer insurgé, en Colombie, ne dure pas seulement en raison de la ferveur combattante, de la rigueur de l'organisation et de l'idéologie des Forces armées révolutionnaires de Colombie. L'argent de la drogue et la pratique des enlèvements y contribuent aussi.

D'autres analystes mettent plutôt en avant les effets de l'obsession néo-conservatrice embourbant en Irak les forces américaines et le projet de réforme du "Grand Moyen-Orient». Mais comment oublier à quel point le Venezuela d'Hugo Chávez devrait compter pour un leadership américain si proche des lobbies pétroliers, et si soucieux de la dépendance des États-Unis envers les importations énergétiques? Au lendemain de la nationalisation des pétroles de la ceinture de l'Orénoque, Christian Stoffaës, président du conseil du Centre d'études prospectives et d'informations internationales, le CEPII, le rappelait : face aux incertitudes du Moyen-Orient, et dans un contexte de hausse des prix du pétrole qui accroît l'intérêt de gisements jugés jusque-là peu attractifs, les États-Unis doivent développer une double stratégie, consistant à assurer les approvisionnements voisins, ceux du Canada et du Mexique, et à exploiter les "nouveaux pétroles d'accès difficile», de l'Arctique à l'Orénoque. Dès lors, «la superpuissance peut espérer, pour demain, inventer à ses portes un nouveau golfe Arabo-Persique qui changera la géopolitique». Encore faut-il que les pays fournisseurs jouent le jeu, à commencer par le Venezuela qui dispose de réserves parmi les plus importantes au monde. Or, rappelle Christian Stoffaës, le Venezuela fut "une des puissances fondatrices de l'OPEP les plus agressives», et c'est bien en Amérique latine qu'est né "le nationalisme pétrolier, au Mexique et au Venezuela, bien avant de faire école» avec un succès tel que les trois quarts des réserves pétrolières mondiales sont aujourd'hui

1 - "George Bush Goes South», The Boston Globe, repris dans l'International Herald Tribune, 10 mars 2007.

2 - Pour un tableau récapitulatif de «l'Amérique latine rebelle», on renvoie au recueil Manières de voir, le Monde diplomatique, publié sous ce titre, $\mathrm{n}^{\circ}$ 90, décembre 2006-janvier 2007. 
contrôlées par des compagnies nationales. Et de conclure: "le Venezuela compte peut-être plus que l'Irak» ${ }^{3} \ldots$

Mais le Venezuela ne compte pas seulement pour son pétrole : il compte tout autant en raison de l'usage qu'Hugo Chávez en fait. Par son style, par son discours, par les moyens que lui procure la manne pétrolière, Chávez donne un relief particulier à deux questions essentielles, indépendamment du jugement qu'on peut porter sur ses pratiques de pouvoir et sur la pertinence de ses choix économiques. La première porte sur les ambiguiités de la consolidation démocratique en Amérique latine et sur les politiques sociales mises en œuvre. La seconde porte sur la dialectique entre indépendance nationale, constructions régionales et ordre mondial. Si ce dossier de Transcontinentales ne s'arrête pas en détail sur le cas vénézuélien, il traite largement de ces deux sujets, déclinés sous diverses formes. Dans chacun des cas, Chávez constitue l'un des pôles autour desquels s'ordonnent les nouvelles dynamiques latino-américaines. Un pôle radical qui, en dépit de la rhétorique du tribun à la chemise rouge, marginalise le castrisme vieillissant. En effet, le mouvement en faveur d'une incertaine «révolution bolivarienne» dispose de moyens propres, pour agir à l'intérieur du pays en donnant le pas aux «conseils communaux» participatifs sur le système représentatif tout en accroissant les pouvoirs présidentiels, comme à l'extérieur où l'assistance fournie aux "pays frères» est considérable. En outre, le Venezuela de Chávez ne subit pas, comme Cuba, le poids d'un blocus américain ( $60 \%$ des exportations de pétrole du pays vont vers les États-Unis), et il se déploie dans un temps qui n'est plus celui de la guerre froide, mais celui d'une Amérique latine s'émancipant de la doxa néo-libérale la plus dure, tout en cherchant sa voie entre mondialisation et multipolarité ${ }^{4}$.

Dans cette nouvelle géométrie, le pôle opposé au radicalisme de Chávez est connu, et d'une certaine façon il est classique : le Mexique de Felipe Calderón et la Colombie d'Álvaro Uribe le définissent, sans que cette droite libérale soit satisfaite en tout de la politique américaine. Un troisième pôle est dessiné, avec des variantes, par une autre gauche, qu'on peut dire, pour faire bref, sociale-démocrate: affichée comme telle au Chili de Michelle Bachelet, plus ambiguë au Brésil de Luiz Inácio Lula da Silva. Bien entendu, l'Amérique latine est trop vaste, trop peuplée (plus de 500 millions $\mathrm{d}^{\prime}$ habitants), trop diverse (23 États, de poids et de niveaux de vie très variables ${ }^{5}$ ) pour qu'on la considère d'un bloc. Mais partout les gauches (et la droite!) y sont confrontées à la question de la gestion du pouvoir, au défi des inégalités, à la quête de la croissance, et aux choix à opérer en matière de coopération régionale.

3 - Christian Stoffaës : «Pétroles de l'Orénoque : l'OPEP est toujours là ", La Tribune, 3 mai 2007. Pour une analyse éclairante sur le Venezuela d'aujourd'hui, voir Frédérique Langue : «Pétrole et révolution dans les Amériques. Les stratégies bolivariennes de Hugo Chávez», Hérodote, n 123, 2006, p. 41-61.

4 - Pour un dossier sur les perspectives cubaines, voir "Cuba, un castrisme sans Fidel ?», Problèmes d'Amérique latine, $\mathrm{n}^{\circ}$ 61/62, automne 2006.

5 - Le PIB moyen par habitant oscille de 6518 dollars au Mexique à 974 dollars en Bolivie. En parité de pouvoir d'achat, l'Argentine (13298 dollars) l'emporte sur le Chili (10874 dollars) et le Mexique (9 803 dollars), la Bolivie restant à la traîne (2 720 dollars). UNDP, Rapport sur le développement humain 2006. 
Cette montée de la gauche latino-américaine, et les enjeux qu'elle nourrit, expliquent pourquoi les regards se tournent avec une acuité nouvelle vers l'Amérique latine. Au-delà des publications spécialisées, telles que les Cahiers des Amériques latines et Problèmes d'Amérique latine, les nombreux numéros spéciaux de revues généralistes en témoignent ${ }^{6}$. Dans l'espace limité qui est le sien, le dossier de ce numéro de Transcontinentales consacré à l'Amérique latine offre des éclairages qui portent tous, à leur façon, sur la dialectique entre pouvoirs, sociétés, croissance et territoires à l'heure de la mondialisation.

L'étude de Jean-Paul Deler et Henri R. Godard est explicite à cet égard. Consacré aux sept pays andins qui s'étendent le long de la cordillère, depuis le Venezuela jusqu'au Chili et à l'Argentine, enrichi de multiples cartes, leur article illustre comment, par-delà des points communs - forte urbanisation, tradition coloniale d'extraversion économique qui reste un marqueur très contemporain - les variations du maillage du territoire traduisent des rapports différents au système mondial autant qu'au pouvoir national. L'existence de pôles structurants majeurs et d'enclaves minières, pétrolières ou militaires mondialisées n'exclut pas la présence de vastes espaces à la traîne, entre autres nombre de terres amérindiennes et les "angles morts des territoires nationaux», ces "périphéries mal contrôlées » propices à l'implantation de l'économie de la drogue ou à la constitution de sanctuaires des guérillas. La forte extraversion économique repose sur les matières premières, et rend donc la croissance vulnérable face aux fluctuations des marchés mondiaux. Elle laisse aussi derrière elle «d'amples poches de marginalisation et d'indigence» que masquent partiellement les indices généralement bons de l'Amérique latine (produit national brut par tête, indice de développement humain) en comparaison de l'ensemble des pays dits « du Sud». Ces deux paramètres posent aux gouvernants des défis essentiels. À l'heure de «l'essoufflement du modèle politique porteur des thèses d'un néolibéralisme économique censé résoudre les nombreux problèmes des pays latino-américains", les auteurs constatent qu'après l'arrivée de la gauche au pouvoir «le cap économique suivi reste le plus souvent néo-libéral, tant sont fortes les contraintes du système économique mondial dominant» et les difficultés à réformer "un système économique dont la dynamique se nourrit des différences de potentiels régionaux et de la permanence d'inégalités socio-spatiales».

On retrouve, dans l'étude d'Olivier Dabène portant sur l'évolution des politiques d'intégration régionale en Amérique latine, le poids des mêmes contraintes. Résumant l'histoire des processus d'intégration depuis les lendemains de la seconde guerre mondiale, l'auteur constate que le modèle planificateur visant au développement, tel qu'élaboré par Raúl Prebisch, premier secrétaire général de la Commission économique pour l'Amérique latine et les Caraïbes (CEPALC), a vite été "soit ignoré dans les années 1950, soit dévoyé dans les années 1960, où il connut une dérive libre-échangiste et un abandon de l'ambition développementaliste». Le tournant

6 - Par exemple : Questions internationales : "Amérique latine», ${ }^{\circ} 18$, mars-avril 2006; Hérodote : "Amérique latine : nouvelle géopolitique», $\mathrm{n}^{\circ} 123,4^{\mathrm{e}}$ trimestre 2006; Géopolitique : "Amérique latine, une ère nouvelle», $\mathrm{n}^{\circ}$ 96, décembre 2006-janvier 2007. 
néo-libéral des années 1980 n'a fait qu'accentuer ces choix, qui ont marqué l'essentiel des accords régionaux ou continentaux, d'essence essentiellement commerciale. L'analyse fine du Mercosur, - «méthode d'intégration médiane» entre les structures de libre-échange inspirées par Washington (ALENA et ZLEA) ${ }^{7}$ et l'Alternative bolivarienne pour les Amériques (ALBA), lancée par Hugo Chávez au nom de «la solidarité entre les peuples» - éclaire bien les ambiguïtés d'une construction régionale tirée par les deux poids lourds de l'Amérique du Sud, le Brésil et l'Argentine. L'amenuisement du rôle intégrationniste des experts du Secrétariat d'assistance technique du Mercosur, les structures programmées du futur Parlement du Mercosur, écartant là encore la dynamique supranationale, et les limites du Fonds de convergence structurelle en matière de réduction des «asymétries de développement entre États membres et entre les régions» témoignent au total d'un bilan mitigé de «la gauche mercosurienne». Une gauche qui tente certes de développer des solidarités, mais qui s'avère «tout autant réticente que la droite face à toute évolution susceptible de faire franchir à l'intégration le seuil fatidique de la supranationalité». Cela ne l'empêche pas de prôner une dose d'interventionnisme étatique au service d'une stratégie de redistribution pragmatique, propre à la gauche réformatrice.

C'est par un autre biais que Virginie Laurent traite du croisement entre question sociale et stratégies politiques: celui de l'indianité. L'élection d'Evo Morales à la présidence de la Bolivie - le pays, il est vrai, compte la plus forte proportion d'Amérindiens - a marqué à cet égard un tournant qui s'inscrit dans ce qu'Yvon Le Bot avait appelé en 2004 "le renversement historique de la question indienne en Amérique latine»: ce passage "du refus à l'affirmation ${ }^{8}$ ", refus d'un système discriminatoire à l'affirmation de soi, au sein même du système. Virginie Laurent analyse comment "la percée électorale indigène» prend corps dans une histoire marquée par de multiples conflits, puis, dans les années 1990, par «l'abandon d'une position abstentionniste souvent défendue face à un système politique jugé fermé", et par "une volonté nouvelle de jouer la carte de la participation par le biais de forces et de leaders qui se démarquent de la classe politique traditionnelle». Elle souligne comment, dans un nouveau rapport à la nation, la "citoyenneté rénovée qui s'applique à tous s'accompagne en outre d'une citoyenneté différente, ratifiée par la validation constitutionnelle du caractère multiethnique et pluriculturel de la Colombie (1991), du Pérou (1993), de la Bolivie (1994) et de l'Équateur (1998)». Cette "conversion de l'indianité en capital politique» n'a rien de mécanique. Elle invoque des paramètres identitaires, mais elle entend sortir aussi de l'enfermement communautaire: le Mouvement vers le socialisme (MAS) d'Evo Morales, en Bolivie, se fonde ainsi sur une idéologie "qui dépasse les seuls intérêts indigènes» et les seuls Indiens, et qui ancre à gauche un projet s'appuyant sur la démocratie participative et sur la souveraineté nationale anti-libérale. Pour autant, l'exercice du pouvoir, à la base d'abord, puis dans les instances nationales, n'est

7 - ALENA : Accord de libre-échange nord-américain, dont le Mexique est partie prenante. ZLEA : Zone de libre-échange des Amériques.

8 - Yvon Le Bot, "Le renversement historique de la question indienne en Amérique Latine», Amérique latine Histoire et Mémoire, $\mathrm{n}^{\circ}$ 10-2004 - Identités: positionnements des groupes indiens en Amérique latine. http://alhim.revues.org/document100.html. Consulté le 6 mai 2007. 
pas sans problème. Si dans toute l'Amérique latine, depuis une décennie, «le pari indien semble gagné sur la scène publique, en termes de visibilité», reste à faire ses preuves, en matière économique et sociale, mais aussi pour gérer au mieux la diversité ethnique. Sous la présidence Morales, en effet, "la polarisation de la société bolivienne» semble s'accentuer.

C'est au Mexique que nous conduit l'étude suivante, de Carlos Alba et Pascal Labazée, qui appelle à repenser la question du secteur informel dans les métropoles des pays pauvres ou des pays émergents. Il est temps, disent les auteurs, d'abandonner les vieux modèles qui analysent le secteur informel en termes "de marginalité, de dépendance, de dualité». Ces concepts sont devenus inopérants. En raison même de la place de ces "micro-activités", au premier chef : ne comptent-elles pas en 2004, au Mexique, pour $45 \%$ des emplois dans les villes de plus de 100000 habitants ? Mais aussi en raison de nouvelles dynamiques qui contribuent à "l'insertion de l'économie populaire dans la mondialisation ». Alba et Labazée soulignent ainsi comment «l'expansion de certains types d'activités, liées à l'expansion de nouveaux circuits marchands internationaux ayant recours à la fraude, est un facteur central dans l'apparition des nouvelles configurations institutionnelles qui accompagnent les politiques de libéralisation». Loin d'être marginale, cette informalité se situe désormais "au coeur des dynamiques productives orientées vers l'extérieur», ce qui ne manque pas de conférer aux ambulants, «signe des difficultés de l'économie ouverte à créer des emplois salariés», un rôle important "dans les luttes politiques locales voire nationales, le plus souvent en tant qu'acteurs dominés dans des systèmes de type clientéliste ou corporatiste». Les déréglementations accompagnant le tournant libéral ont affaibli «les institutions étatiques de contrôle et de répression de l'illégalité», alors même que se développent "les réseaux de contrefaçons et de contrebande», parfois très sophistiqués, et que déclinent les petites activités traditionnelles de production. On ne peut donc s'étonner de constater que «la mise en place de nouvelles institutions et le vote de nouvelles lois répressives se heurtent au poids économique des réseaux illégaux, et aux enjeux sociaux et politiques dans lesquels ils sont parvenus à s'insérer».

Dernière étude de ce dossier, sur une autre facette - inattendue - de la mondialisation, l'article de l'arabisant Alain Roussillon se penche sur les diasporas arabes et musulmanes établies en Amérique latine, telles que les montre une série de documentaires commandités en 2005 à des cinéastes égyptiens par la chaîne de référence du monde arabe, Al-Jazeera. L'émigration du Proche-Orient vers l'Amérique latine n'est certes pas récente, comme l'avaient montré les récits de vie collectés par Sélim Abou dans son classique Liban déraciné. Immigrés dans l'autre Amérique, publié dans la collection "Terre humaine» dans les années 1970. Ce qui est neuf tient plus à la circulation du regard et de la parole, à cette boucle quasi cybernétique - au sens de Norbert Wiener et de Gregory Bateson - par laquelle l'information suscitée par l'initiative de la télévision qatarie, et fournie par la diaspora arabe en Amérique latine, ne parle pas seulement de celle-ci, mais renvoie aussi un message à la terre d'origine. Au passage, on mesure combien cette diaspora souvent qualifiée de turcos a su s'intégrer dans son nouveau monde, et parvenir dans bien des cas au gotha des affaires et de la vie professionnelle, voire au sommet de la vie politique (Carlos 
Menem en Argentine, Abdalla Bukaram et Jamil Moawwad - ou Mahuad - en Équateur, Julio Caesar Turbay Ayala en Colombie...). Certes, en donnant à voir des «Latino-Arabes» ou des "latino-musulmans» qui ont réussi dans tous les domaines, cette série de documentaires laisse dans l'ombre les petits émigrés. Mais, nous dit Alain Roussillon, il s'agit aussi, implicitement ou explicitement, d'offrir "des illustrations emblématiques des accomplissements dont sont capables les Arabes quand les circonstances s'y prêtent», femmes incluses. Pourtant, des blessures perceraientelles? Quand l'intégration invoquée se fait au prix de l'abandon fréquent de la langue arabe, non transmise aux enfants; ou quand l'appartenance à l'islam, une fois "passées les protestation de coexistence pacifique», s'opère dans "une manière de retranchement», pour mieux le conserver "inentamé, à distance de la société, sans interaction avec elle». Ce qui n'exclut pas, en contrepoint, une nouvelle "effervescence identitaire». Et Roussillon de se demander si un message subliminal de la série d'Al-Jazeera n'est pas "que l'âge d'or de l'émigration vers l'Amérique latine est bel et bien passé et que les Arabes qui en tentent aujourd'hui l'aventure risquent d'être poursuivis par leur identité proche-orientale, 11 septembre 2001 oblige...»

L'entretien avec Hélgio Trindade, politologue et ancien recteur de l'université fédérale de Porto Alegre, complète ce dossier par un large tour d'horizon, où le Brésil prend toute sa place. Rappelant l'histoire des populismes et des régimes autoritaires en Amérique latine, Hélgio Trindade nous met en garde contre l'usage abusif du concept de populisme dont la mise en avant, aujourd'hui, "tend à appauvrir la richesse des changements qui définissent l'apport actuel de l'Amérique latine à la politique mondiale». Il éclaire comment le champ d'innovation que fut Porto Alegre, des expériences de budget participatif à la naissance du Forum social mondial, s'est trouvé en quelque sorte dépassé quand «l'accession du Parti des travailleurs à des gouvernements d'États puis au pouvoir fédéral a déplacé l'axe politique des avancées démocratiques». Avec l'élection, et surtout la réélection triomphale de Lula à la présidence de la République en 2006, le Parti des travailleurs suit moins la voie radicale (une minorité critique a fait scission) qu'il ne cherche à consolider la démocratie. Comment? En s'efforçant, nous dit Trindade, de combiner prudence financière, croissance économique, priorité à l'éducation et politiques sociales, et en élargissant la base politique et sociale du régime, une stratégie impérative, du reste, dans un contexte de gouvernement de coalition. Conservant du passé une part de libéralisme, sans renier le rôle de l'État développementaliste, Lula entend promouvoir la croissance en maîtrisant l'inflation, en remboursant la dette, et en définissant une double stratégie "d'expansion de l'économie, appuyée sur les potentialités du marché intérieur stimulées par les plans coordonnés par l'État, et sur une politique d'insertion internationale "souveraine" dans la globalisation". Cette quête de souveraineté dans l'ouverture explique pourquoi le Brésil fut l'un des piliers du Groupe des 21, qui contribua en 2003 à bloquer les négociations de l'OMC à Cancún, face aux politiques agricoles protectionnistes des États-Unis et de l'Union européenne; pourquoi, avec l'Inde, Brasília plaide pour l'élargissement du nombre des membres permanents du Conseil de sécurité; et pourquoi, en avril 2007, le Brésil entend réfléchir avec d'autres États latino-américains à la possible constitution d'une «Banque du Sud» voulue par Hugo Chávez, qui vient de quitter la Banque mondiale et le Fonds monétaire international. Ceci n'a pas 
empêché Lula de recevoir GeorgeW. Bush dans sa tournée latino-américaine. Pays émergent, le Brésil entend jouer ses cartes avec un certain pragmatisme qu'autorise, sur le plan intérieur comme sur le plan international, le satisfecit donné par l'électorat au charismatique dirigeant syndical devenu une personnalité mondiale.

\section{Variations}

Loin de l'Amérique latine, mais centrée aussi sur la question démocratique, l'étude de Stéphanie Tawa Lama-Rewal consacrée aux associations de résidents à New Delhi illustre toute l'ambiguïté du terme de «démocratie locale». Qu'observe-t-elle dans la capitale indienne (13 millions d'habitants en 2001)? Le poids croissant de la classe moyenne possédante, celle qui dispose du plus d'entregent pour faire entendre la voix des propriétaires résidant le plus souvent dans des lotissements de qualité. Le gouvernement célèbre cette pratique de "partenariat» avec les associations comme un outil de bonne gouvernance. Les opposants y voient à l'inverse non seulement un outil paradoxal qui réussit à réduire le poids des élus locaux, en partie court-circuités par un dialogue directement conduit entre résidents et administration, mais aussi une pratique électoraliste, dans la mesure où se constituent des réseaux clientélistes. L'auteur, quant à elle, analyse le «discours autolégitimant» de cette classe moyenne aisée qui se définit comme portant la voix des «citoyens honnêtes", contre les «bureaucrates corrompus» et contre les fraudeurs occupant sans autorisation des terrains publics, à commencer par les habitants des bidonvilles. Les associations de résidents, et leurs puissantes fédérations, réussissent à infléchir des décisions ou des projets gouvernementaux (schéma d'urbanisme, modalités de la privatisation de la distribution d'eau et de l'électricité, calcul de l'impôt foncier). Elles témoignent donc de l'efficacité des "procédures participatives dans la gouvernance urbaine», mais au profit d'une section limitée de la population. En marginalisant «la dimension représentative de la démocratie locale», ces procédures ont «des conséquences tragiques pour les pauvres» dont le mode d'usage du sol urbain se trouve privé de légitimité. Reste à voir si un projet de «loi de participation communautaire», structuré autour d'un maillage fin d'assemblées de quartiers nommées par les élus, permettra aux acteurs politiques locaux de mieux contrôler une démocratie participative redéfinie sur une base sociologique beaucoup plus ouverte.

La dernière étude nous ramène à la question identitaire, telle qu'elle prend forme dans l'histoire des musées construits à Taiwan depuis un siècle. La mise en scène de la mémoire, dans une île qui connut une histoire tourmentée, traduit les mutations d'un imaginaire officiel, qui célébra tour à tour la présence japonaise, le nationalisme chinois porté par le Kuomintang, le nouveau nationalisme taiwanais, mais aussi, in fine, la reconnaissance des minorités autochtones. Avec précision, Lee Wei-i dénoue le fil des constructions patrimoniales, éclaire la portée de leur style et de leur lieu d'implantation, la figure des décideurs, le message transmis par les expositions phares. Le Musée national d'histoire offre non seulement «une reconstruction romantique de l'histoire de la Chine antique», mais aussi la célébration "de l'histoire d'un état nationaliste moderne». La "salle des grands hommes» reconstruit ainsi "une généalogie des ancêtres constitutifs de la nation chinoise», 
des sages antiques (Confucius bien sûr) aux héros des temps modernes (Sun Yat-sen au premier chef) en passant par les grands empereurs Han et Tang. Quand Taiwan ne peut plus prétendre représenter la Chine, désormais entrée à l'ONU, une autre logique prévaut, qu'appuiera pour partie une politique d'expositions internationales. D'autres musées fabriquent alors «un imaginaire de la modernité en phase avec son temps, que ce soit un imaginaire national en défense (malgré tout) de la légitimité politique du régime de Taipei, ou un imaginaire local fondé sur le vécu de la société taiwanaise». Six vagues de constructions se succèdent ainsi en un siècle, portant chacune une image différente de l'identité, du patrimoine, et de la nation.

L'histoire, on le sait, est en constante reconstruction. Nous en avons ici comme un avant-goût, alors que se prépare le premier colloque du réseau des instituts français de recherche à l'étranger qui explorera, à l'automne 2007, la "présence du passé", en étudiant sous de multiples approches les formes contemporaines de la dialectique entre mémoire et sociétés.

Jean-Luc Racine 